\title{
Analytic Gradients for Restricted Active Space Second-order Perturbation Theory
} (RASPT2)

Yoshio Nishimoto ${ }^{1, a)}$

Graduate School of Science, Kyoto University, Kyoto 606-8502, Japan

(Dated: 3 May 2021)

The computational cost of analytic derivatives in multireference perturbation theory is strongly affected by the size of the active space employed in the reference self-consistent field calculation. To overcome previous limits on active space size, the analytic gradients of single-state restricted active space second-order perturbation theory (RASPT2) and its complete active space variant (CASPT2) have been developed and implemented in a local version of OpenMolcas. Similar to previous implementations of CASPT2, the RASPT2 implementation employs the Lagrangian or Z-vector method. The numerical results show that restricted active spaces with up to 20 electrons in 20 orbitals can now be employed for geometry optimizations.

a)Electronic mail: nishimoto@kuchem.kyoto-u.ac.jp. 


\section{INTRODUCTION}

Accurate and efficient quantum chemical approaches are indispensable for predicting electronic and geometrical properties. For this purpose, electron correlation must be considered to the greatest extent possible, ideally with a low computational cost. One useful and well-known approach to account for electron correlation is multiconfiguration self-consistent field (MCSCF) calculations followed by electron excitation, namely multireference (MR) or post-MCSCF treatments, to consider dynamic and static (nondynamic) electron correlation in a balanced way. MR methods include MR coupled-cluster ${ }^{1-3}$ and configuration interaction ${ }^{4}$ approaches. However, MR perturbation theory (MRPT) may be the most balanced approach in terms of computational cost and accuracy. The most well-known MRPT is probably the complete active space second-order perturbation theory (CASPT2).$^{5-7}$ Other MRPTs such as (extended) ${ }^{8}$ multiconfiguration quasidegenerate second-order perturbation theory [(X)MCQDPT2], ${ }^{9} n$-electron valence state secondorder perturbation theory (NEVPT2), ${ }^{10-12}$ generalized van Vleck second-order perturbation theory (GVVPT2), ${ }^{13}$ and retaining the excitation degree perturbation theory (REPT) ${ }^{14}$ have also been employed for various tasks.

Recent efforts to develop analytic derivatives of MRPTs ${ }^{15}$ have made it possible to efficiently compute properties. Earlier studies reported such implementations in MOLPRO, ${ }^{16}$ BAGEL, ${ }^{17}$ GAMESS-US, ${ }^{18}$ and TeraChem ${ }^{19}$ for different MRPT methods. However, the size of the active space in these past works was rather limited. To the best of our knowledge, the largest active space in terms of the number of determinants [or configuration state functions (CSFs)] applied in analytic derivatives corresponds to 12 electrons in 12 orbitals $(12 \mathrm{e}, 12 \mathrm{o}){ }^{18}$ or $(12 \mathrm{e}, 11 \mathrm{o}) .{ }^{20,21}$ In the former calculation, the number of determinants was 853,776 in GAMESS-US.

This severe limitation on active space size is due to the full configuration interaction (CI), or complete active space (CAS), treatment in the active space, and the limitation is even more severe for subsequent post-CASSCF approaches. The formal computational cost of CAS treatment grows as a factorial, and higher-order reduced density matrices are needed in post-CASSCF calculations if the internal contraction scheme is applied. The situation is worse for analytic derivatives of MRPTs because one has to contract terms that formally scale as $N_{\mathrm{CSF}} N_{\mathrm{act}}^{6}$ to $N_{\mathrm{CSF}} N_{\mathrm{act}}^{8}$ (a power of eight for NEVPT2), where $N_{\mathrm{CSF}}$ and $N_{\text {act }}$ are the number of CSFs (or determinants) and the number of active orbitals, respectively. Thus, one must carefully develop a computationally and memory efficient algorithm ${ }^{20}$ to allow larger active spaces. 
One solution to this factorial growth is to employ restricted active space (RAS) ${ }^{22}$ SCF references followed by perturbation theory (i.e., RASPT2). ${ }^{23,24}$ In RASSCF, the active space is first partitioned into three subspaces: RAS1, RAS2, and RAS3. The RAS2 space is treated as the CAS space, and full CI is performed. In RAS1 and RAS3, the numbers of holes and electrons, respectively, are limited by setting maximum values. As a result, the number of CSFs or determinants for the RAS is much smaller than that for the corresponding CAS. Using this approach, an initial RASPT2 study was conducted with an active space as large as $(28 \mathrm{e}, 32 \mathrm{o}) .{ }^{24}$ Similar approximate CAS treatments for MRPTs have been developed for MCQDPT, including the quasi-CAS QDPT, ${ }^{25}$ general MCQDPT, ${ }^{26}$ and occupation-restricted multiple active space (ORMAS) PT $^{27}$ methods. One important difference between these QDPT-based methods and RASPT2 is the inclusion of the fully internal excitations (perturbative two-electron excitations within the active space): to date, developed RASPT2 methods do not consider these excitations, primarily because of the complications resulting from the internal contraction. The RASPT2 implementation by Celani et al. in MOLPRO ${ }^{23}$ includes the fully internal excitations, but this excitation class is not internally contracted. Another famous CAS-based approach combines the density-matrix renormalization group (DMRG) with MRPT (DMRG-CASPT2). ${ }^{28}$

This paper describes the development of analytic gradients for fully internally contracted single-state RASPT2 along with CASPT2. The developed method is applied to typical $\pi$-rich molecules, and calculations are performed with active spaces containing up to 20 electrons in 20 orbitals.

\section{METHODS}

CASSCF and CASPT2 are the special cases of RASSCF and RASPT2, respectively. Thus, here, we focus on RASSCF and RASPT2 as general cases. In this section, $p, q, r$, and $s$ refer to general orbitals, $i$ and $j$ refer to inactive (doubly occupied) orbitals, $t$ and $u$ refer to active orbitals, $a$ and $b$ refer to secondary (virtual) orbitals, and $\phi$ and $\chi$ refer to internally contracted bases.

This section does not provide the full details of algorithm development; rather, it provides an outline of the algorithm and highlights a few differences between the analytic derivatives of CASPT2 and RASPT2 because many equations are equivalent to those presented in earlier studies for CASPT2. Readers interested in the details should refer to, for instance, Refs. 16, 17, 19, and 29. 


\section{A. RASPT2 Energy}

In RASPT2, the zeroth-order Hamiltonian is defined by

$$
\hat{H}^{(0)}=\hat{P} \hat{F} \hat{P}+\hat{Q} \hat{F} \hat{Q}
$$

where $\hat{P}=|0\rangle\langle 0|(|0\rangle$ is a reference state obtained by SCF $)$ is the projector on the reference space, and $\hat{Q}$ is the complementary projector. The Fock operator is defined by

$$
\hat{F}=\sum_{p q} f_{p q} \hat{E}_{p q}
$$

where $\hat{E}$ is the one-electron spin-averaged excitation operator, and $f_{p q}$ is the Fock matrix:

$$
f_{p q}=h_{p q}+\sum_{r s}\left((p q \mid r s)-\frac{1}{2}(p r \mid q s)\right) D_{r s}^{\mathrm{SA}}
$$

with the state-averaged one-electron density matrix $D_{r s}^{S A}$. Analyzing the structure of the Fock operator [Eq. (2)] shows that it consists of 25 blocks generated by the combinations of inactive, active (RAS1, RAS2, and RAS3), and external orbitals for RASPT2 (or nine for CASPT2).

The (non-variational) second-order perturbation energy $E^{(\mathrm{PT} 2)}$ is then obtained as a minimum of the Hylleraas functional

$$
E^{(\mathrm{PT} 2)} \stackrel{\min }{=} E_{2}=2\left\langle\Psi^{(1)}|\hat{H}| \Psi^{(0)}\right\rangle+\left\langle\Psi^{(1)}\left|\hat{H}^{(0)}-E^{(0)}+E_{\text {shift }}\right| \Psi^{(1)}\right\rangle .
$$

The zeroth-order wavefunction $\left|\Psi^{(0)}\right\rangle$ is obtained at the SCF level, and the first-order wavefunction $\left|\Psi^{(1)}\right\rangle$ is generally defined by operating two-electron excitations: ${ }^{24}$

$$
\left|\Psi^{(1)}\right\rangle=\sum_{p q r s} T_{p q r s} \hat{E}_{p q r s}\left|\Psi^{(0)}\right\rangle
$$

In Eq. 5, $T_{p q r s}$ is the amplitude of the excitation, which is obtained by solving the amplitude equation

$$
\frac{1}{2} \frac{\partial E_{2}}{\partial T_{\text {pqrs }}}=\left\langle\Phi_{\text {pqrs }}|\hat{H}| \Psi^{(0)}\right\rangle+\left\langle\Phi_{\text {pqrs }}\left|\hat{H}^{(0)}-E^{(0)}+E_{\text {shift }}\right| \Psi^{(1)}\right\rangle=0,
$$

where $\left|\Phi_{\text {pqrs }}\right\rangle$ is the doubly excited configuration. This equation is solved iteratively. In Eqs. (4) and (6), the real ${ }^{30}$ and imaginary ${ }^{31}$ level-shift parameter values are collectively represented as $E_{\text {shift }}$, which can be expressed as ${ }^{30,31}$

$$
E_{\text {shift }}=E_{\text {shift }}^{\text {real }}+\frac{\left(E_{\text {shift }}^{\text {imaginary }}\right)^{2}}{\hat{H}_{\mathrm{D}}^{(0)}-E^{(0)}}
$$


where $E_{\text {shift }}^{\text {real }}$ and $E_{\text {shift }}^{\text {imaginary }}$ are provided as parameters and cannot be non-zero simultaneously, and $\hat{H}_{\mathrm{D}}^{(0)}$ is the diagonal part of $\hat{H}^{(0)}$. The RASPT2 energy is finally obtained as a sum of the RASSCF and PT2 energies: $E^{\mathrm{RASPT} 2}=E^{\mathrm{RASSCF}}+E^{\mathrm{PT} 2}$, where the unshifted second-order perturbation energy $E^{\mathrm{PT} 2}$ is

$$
E^{\mathrm{PT} 2}=2\left\langle\Psi^{(1)}|\hat{H}| \Psi^{(0)}\right\rangle+\left\langle\Psi^{(1)}\left|\hat{H}^{(0)}-E^{(0)}\right| \Psi^{(1)}\right\rangle
$$

$E^{\mathrm{PT} 2}$ coincides with $E^{(\mathrm{PT} 2)}$ only when both the real and imaginary level-shift values are zero. Strictly speaking, above equations must be formulated with indices of the internally contracted basis and distinguish singlet and triplet excitations, making this method more complicated. For further discussion, readers should refer to, for instance, Refs. 6, 7, and 32.

\section{B. First-order Derivatives}

Since the RASPT2 energies are not variational with respect to changes in wavefunction parameters, analytic derivatives of the energies are needed to evaluation of the response (derivative) of the wavefunction parameters. As in earlier studies, the first-order derivatives of the RASPT2 energies in this study were evaluated using the Lagrangian approach. ${ }^{33}$ At the first order, the equations derived using this approach are essentially equivalent to those derived by the Z-vector method. ${ }^{34}$

First, we define the Lagrangian, which can be written as a sum of the RASSCF and the PT2 Lagrangians:

$$
\mathscr{L}^{\mathrm{RASPT} 2}=\mathscr{L}^{\mathrm{RASSCF}}+\mathscr{L}^{\mathrm{PT} 2}
$$

The RASSCF Lagrangian $\mathscr{L}^{\text {RASSCF}}$ is defined as the sum of the RASSCF energy $\left(E^{\text {RASSCF}}\right)$ and the constraint conditions imposed when solving the RASSCF equation:

$$
\begin{aligned}
\mathscr{L}^{\mathrm{RASSCF}} & =E^{\mathrm{RASSCF}}+\frac{1}{2} \operatorname{Tr}\left[\mathbf{Z}\left(\mathbf{A}-\mathbf{A}^{\dagger}\right)\right]-\frac{1}{2} \operatorname{Tr}[\mathbf{X}(\mathbf{S}-\mathbf{I})] \\
& +\sum_{N} \omega_{N}\left[\sum_{I} z_{I, N}\left\langle I\left|\hat{H}-E_{N}^{\mathrm{ref}}\right| N\right\rangle-\frac{1}{2} x_{N}(\langle N \mid N\rangle-1)\right],
\end{aligned}
$$

where the second term is the generalized Brillouin condition with the orbital gradient $\mathbf{A}-\mathbf{A}^{\dagger},{ }^{16,35}$ the third term is the requirement for the orthonormalization of molecular orbitals (MOs) with the overlap matrix $\mathbf{S}$ in the MO basis, and the fourth term is the CI condition. In the fourth term, $\omega_{N}$ is the weight in state averaging, $E_{N}^{\text {ref }}$ is the RASSCF energy relevant to CI for state $N$, and $I$ is the CSF index. The Lagrangian multipliers $(\mathbf{Z}, \mathbf{X}, \mathbf{z}$, and $\mathbf{x})$ are determined by solving the following 
simultaneous equation, which is usually referred to as the Z-vector: ${ }^{34}$

$$
\left\{\begin{array}{l}
\frac{\partial \mathscr{L}^{\mathrm{RASSCF}}}{\partial \kappa_{p q}}=0 \\
\frac{\partial \mathscr{L}^{\mathrm{RASSCF}}}{\partial c_{I, N}}=0
\end{array},\right.
$$

where $\kappa_{p q}$ is the orbital rotation parameter, and $c_{I, N}$ is the CI coefficient. Details of the Z-vector or coupled-perturbed MCSCF equation can be found, for instance, in Refs. 36 and 37. Once the Lagrangian multipliers are determined, one can compute the gradient of the energy as the partial derivative of the Lagrangian:

$$
\frac{\mathrm{d} E^{\mathrm{RASSCF}}}{\mathrm{d} \alpha}=\frac{\partial \mathscr{L}^{\mathrm{RASSCF}}}{\partial \alpha}
$$

where $\alpha$ is the derivative parameter (nuclear coordinates). If the single state is employed, the first-order derivatives can be computed without solving the response equation.

The RASPT2 Lagrangian can be written as it follows:

$$
\begin{aligned}
\mathscr{L}^{\mathrm{PT} 2} & =\sum_{s} \mathscr{L}_{s}^{\mathrm{PT} 2} \\
& +\sum_{\text {pqrs }} \lambda_{\text {pqrs }}\left(\left\langle\Phi_{\text {pqrs }}|\hat{H}| \Psi^{(0)}\right\rangle+\left\langle\Phi_{\text {pqrs }}\left|\hat{H}^{(0)}-E^{(0)}+E_{\text {shift }}\right| \Psi^{(1)}\right\rangle\right) \\
& +\sum_{i}^{\text {core inactive }} \sum_{j}^{\mathrm{c}} Z_{i j}^{\mathrm{c}} f_{i j} .
\end{aligned}
$$

The second term in Eq. (13) is the variational condition of the amplitude [Eq. (6)] with multiplier $\lambda_{\text {pqrs }}$, and the third term is required for the frozen core approximation. $\mathscr{L}_{s}^{\mathrm{PT} 2}$ is the Lagrangian for the excitation class $s$, which corresponds to Eqs. (1a), (1b), .., and (1h) in Ref. 6 and is also defined by

$$
\mathscr{L}_{s}^{\mathrm{PT} 2}=E_{s}^{\mathrm{PT} 2}-\sum_{\phi \chi} \xi_{\phi \chi}\left(\Lambda_{\phi \chi}^{\mathrm{S}}-\mathbf{I}\right)
$$

where $E_{s}^{\mathrm{PT} 2}$ is the perturbation energy from the excitation class $s\left(E^{\mathrm{PT} 2}=\sum_{s} E_{s}^{\mathrm{PT} 2}\right)$, and $\Lambda_{\phi \chi}^{\mathrm{S}}$ corresponds to Eq. (16) in Ref. 7 and is due to the orthonormalized overlap matrix in the internally contracted basis. An additional Lagrangian multiplier $\xi_{\phi \chi}$ is introduced, and it can be computed without iteration.

If the real or imaginary level-shift is employed, the unshifted second-order perturbation energy is no longer variational with respect to changes in the amplitude, because $\frac{\partial E^{(\mathrm{PT} 2)}}{\partial T_{p q r s}}=0$ but $\frac{\partial E^{\mathrm{PT} 2}}{\partial T_{p q r s}} \neq 0$. This non-variational property formally requires the evaluation of the derivative of the 
amplitude, but this evaluation can be avoided by solving a $\lambda$-equation, as in Refs. 19, 29, and 35, to determine the Lagrange multiplier $\lambda_{\text {pqrs }}$ :

$$
\begin{aligned}
\frac{\partial \mathscr{L}^{\mathrm{PT} 2}}{\partial T_{\text {pqrs }}}= & 2\left(\left\langle\Phi_{\text {pqrs }}|\hat{H}| \Psi^{(0)}\right\rangle+\left\langle\Phi_{\text {pqrs }}\left|\hat{H}^{(0)}-E^{(0)}\right| \Psi^{(1)}\right\rangle\right) \\
& +\left\langle\Phi_{\text {pqrs }}\left|\hat{H}^{(0)}-E^{(0)}+E_{\text {shift }}\right| \widetilde{\Psi}^{(1)}\right\rangle=0
\end{aligned}
$$

where $\left|\widetilde{\Psi}^{(1)}\right\rangle=\sum_{p q r s} \lambda_{p q r s}\left|\Phi_{p q r s}\right\rangle$. This $\lambda$-equation is similar in structure to the amplitude equation [Eq. (6)], so it can be iteratively solved by following a similar procedure to the energy calculation.

Since the Z-vector equation has already been implemented in OpenMolcas both without ${ }^{37}$ and with $^{38}$ the density-fitting approximation, the main task of this work is to construct the RASPT2 part of the "source term" 39 of the Z-vector equation:

$$
\begin{aligned}
Y_{p q} & :=\frac{\partial \mathscr{L}^{\mathrm{PT} 2}}{\partial \kappa_{p q}} \\
y_{I, N} & :=\frac{\partial \mathscr{L}^{\mathrm{PT} 2}}{\partial c_{I, N}},
\end{aligned}
$$

which are roughly equivalent to the "orbital Lagrangian" and the "configuration Lagrangian," respectively, in the author's previous studies. ${ }^{18,40}$ Once all the Lagrangian multipliers are determined by solving the Z-vector equation, as in the case of the state-averaged RASSCF [Eq. (11) but where $\mathscr{L}^{\mathrm{RASSCF}}$ is replaced with $\mathscr{L}^{\mathrm{RASPT}}$ ], the derivative of the RASPT2 energy can be evaluated by

$$
\frac{\mathrm{d} E^{\mathrm{RASPT} 2}}{\mathrm{~d} \alpha}=\frac{\partial \mathscr{L}^{\mathrm{RASPT} 2}}{\partial \alpha} .
$$

$Z_{i j}^{\mathrm{c}}$ can be easily computed as ${ }^{41}$

$$
Z_{i j}^{\mathrm{c}}=-\frac{1}{2} \frac{Y_{i j}-Y_{j i}}{f_{i i}-f_{j j}} .
$$

As stated earlier, most of the above equations are equivalent to those derived in previous developments of CASPT2. ${ }^{16,17,19,29}$ One major difference between the CAS and RAS references is the definition of the independent orbital rotations. Independent orbital rotations change the (electronic) energy. When the CAS reference is employed, the independent orbital rotation consists of three subspaces: inactive-active, inactive-external, and active-external blocks. In contrast, for the RAS reference, we need to consider three additional blocks: RAS1-RAS2, RAS1-RAS3, and RAS2-RAS3 blocks. The orbital rotation parameters in these additional blocks are optimized by solving the Z-vector equation. The difference is schematically explained in Fig. 1. The area with diagonal lines in white squares represents the independent orbital rotations for CASPT2. For 


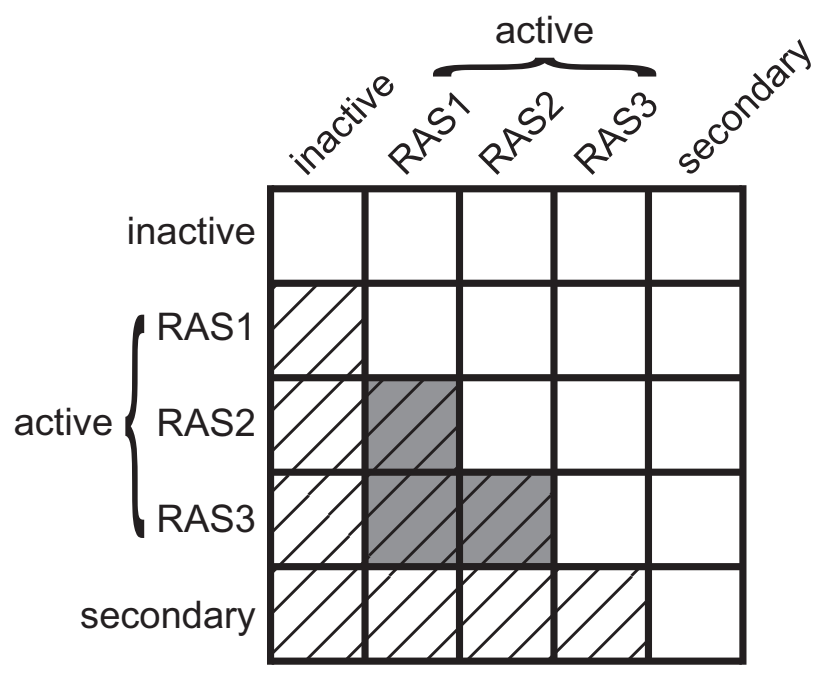

FIG. 1. Independent orbital rotations for CASPT2 and RASPT2.

RASPT2, three additional blocks (gray areas) are considered. The other blank areas are either dependent or redundant (diagonal or off-diagonal blocks, respectively) orbital rotations. The density in the dependent part is constructed with the non-canonical approach ${ }^{42,43}$ because RASPT2 is an invariant theory with respect to rotations within each orbital block. An alternative approach for the active part (iterative) is outlined in Ref. 19.

\section{Implementations}

The above equations, particularly Eqs. (16) and (17), were implemented in a local version of OpenMolcas. ${ }^{44,45}$ Although these equations are formulated based on the Lagrangian method, the actual implementation closely follows that of the equations derived by direct differentiation, as in Refs. 18 and 40. The present implementation can employ the frozen core approximation (i.e., the chemically inert orbitals are neglected) and consider the real and imaginary level-shift. Electron-repulsion (two-electron) integrals can be evaluated conventionally or with the densityfitting approximation ${ }^{38}$ but not with the Cholesky decomposition. The diagonal preconditioning for the active-active rotations in the Z-vector has not been implemented; thus, it was implemented according to Eq. (C.12e) in Ref. 46.

If several states are averaged in the reference SCF calculation, the Fock operator may be defined using either the state-averaged or (unrelaxed) state-specific density matrices and molecular orbitals by canonicalizing the Fock matrix. By default, OpenMolcas employs the state-specific density 
matrix (except for XMS-CASPT2); however, the present implementation for analytic gradients is limited to the state-averaged density matrix [Eq. (3)]. As presented in Refs. 47 and 48, the effect of this difference can sometimes be as large as $0.4-0.5 \mathrm{eV}$. However, the use of the state-specific density matrix can provide an additional source of non-invariance in multistate MRPTs $;{ }^{8,49}$ hence, the use of the state-averaged density matrix is likely favorable even at single-state MRPT levels for geometry optimizations.

\section{COMPUTATIONAL DETAILS}

All calculations were performed with a locally modified version of OpenMolcas. ${ }^{44,45}$ The frozen core approximation was always employed in the perturbation calculation. The cc-pVDZ basis set ${ }^{50,51}$ and the density-fitting approximation with the corresponding RI fitting auxiliary basis set taken from the EMSL basis set exchange $\mathrm{e}^{52-54}$ were employed unless otherwise noted. The ionization potential-electron affinity shift ${ }^{55}$ was set to zero. No symmetry constraints were applied. A single node of a six-core E5-2643 v4 3.40-GHz processor with 48 GB of random-access memory was used to perform all calculations and measure all computational times.

It is useful to define the RAS. In this study, the nomenclature in Ref. 24 is employed: $(i \mathrm{e}, j \mathrm{o}) /(k \mathrm{e}, l \mathrm{o}) / m$, where $i$ and $j$ are the numbers of electrons and orbitals in the entire active space (RAS1+RAS2+RAS3), respectively; $k$ and $l$ are those in the RAS2 space, respectively; and $m$ is the maximum number of electrons excited from RAS1 or into RAS3. Different from the nomenclature in Ref. 24, the definition of the RAS2 space is explicitly written, even if $k$ and $l$ are zero. In this study, the numbers of MOs in RAS1 and RAS3 are always equal.

First, the accuracy of the implemented gradient is discussed using 1,3-butadiene and naphthalene optimized at the Hartree-Fock/cc-pVDZ level of theory. The five-point stencil method with displacement parameters of $1.0 \times 10^{-2}$ and $5.0 \times 10^{-2}$ bohr for 1,3-butadiene and naphthalene, respectively were employed to evaluate the numerical gradients. The lowest two states were averaged in all calculations.

Second, CASPT2 and RASPT2 with various active spaces were applied to trans-1,3,5,7,9,11dodecahexaene $\left(\mathrm{C}_{12} \mathrm{H}_{14}\right)$. The active space consisted of 12 electrons in $12 \pi$ orbitals $(12 \mathrm{e}, 12 \mathrm{o})$ in all calculations. The lowest two states were averaged with an imaginary shift of $0.2 \mathrm{i}$.

Finally, the developed methodology, RASPT2, was applied to dibenzopentalene derivatives 2a and $4 \mathbf{a}$ in Ref. 56. As these two molecules have 16 and 20 electrons in the corresponding $\pi$ 
orbitals, respectively, only RASPT2 calculations were performed, although it would be possible to employ small CASs. RAS $(16 \mathrm{e}, 16 \mathrm{o}) /(4 \mathrm{e}, 4 \mathrm{o}) / 2$ and $\mathrm{RAS}(20 \mathrm{e}, 20 \mathrm{o}) /(4 \mathrm{e}, 4 \mathrm{o}) / 2$ were employed for 2a and 4a, respectively. The lowest three states were averaged with an imaginary shift of $0.2 \mathrm{i}$.

The optimized coordinates of the trans-1,3,5,7,9,11-dodecahexaene and dibenzopentalene derivatives are provided in the supplementary material.

\section{RESULTS AND DISCUSSION}

\section{A. Accuracy of the Implemented Gradient}

First, the accuracies of the implementations with CASPT2 and RASPT2 gradients are briefly presented by comparing the analytic and numerical (with a five-point stencil) gradients. Table I shows that the difference between the gradients is at most $1.0 \times 10^{-5}$ a.u./bohr, indicating the implemented gradients are sufficiently accurate. Here, three active spaces were employed for both species: $\operatorname{CAS}(4 \mathrm{e}, 4 \mathrm{o}), \operatorname{RAS}(4 \mathrm{e}, 4 \mathrm{o}) /(0 \mathrm{e}, 0 \mathrm{o}) / 2$, and $\mathrm{RAS}(4 \mathrm{e}, 4 \mathrm{o}) /(2 \mathrm{e}, 2 \mathrm{o}) / 1$ for 1,3-butadiene and CAS(10e,10o), RAS(10e,10o)/(0e,0o)/2, and RAS(10e,10o)/(4e,4o)/2 for naphthalene. The differences with the different active spaces are reasonably small, indicating that any active space may be employed so long as the reference RASSCF calculation converges. The use of the imaginary shift technique does not degrade the accuracy. Although analytic gradients with the real level-shift can be evaluated with the present implementation, they were not employed in this study. The accuracy for $S_{1}$ is similar to that for $S_{0}$.

Note that it is not possible to perform gradient calculations analytically with the present implementation using, for instance, $\operatorname{RAS}(4 \mathrm{e}, 4 \mathrm{o}) /(2 \mathrm{e}, 2 \mathrm{o}) / 2$ and $\operatorname{RAS}(4 \mathrm{e}, 4 \mathrm{o}) /(0 \mathrm{e}, 0 \mathrm{o}) / 4$ for 1,3-butadiene or $\operatorname{RAS}(10 \mathrm{e}, 10 \mathrm{o}) /(8 \mathrm{e}, 8 \mathrm{o}) / 2$ for naphthalene, which span the same variational space as CAS. In such a case, some of the orbital rotation parameters are linearly dependent on the configuration parameters; thus, the $\mathrm{Z}$-vector equation does not converge. It may be possible to eliminate the linear dependency by eliminating orbital rotations, ${ }^{57}$ but one should perform the CAS calculation. Even though these quasi-complete RASs yield the same energy as the corresponding CAS at the SCF level, they do not yield the same perturbation energy, because the orbitals in the active space are canonicalized in each RAS; hence, the RASs yield a different set of canonical orbitals than the corresponding CAS. 


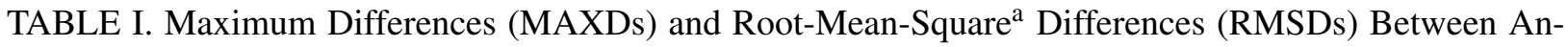
alytic and Numerical Gradients in a.u./bohr for the Ground State at the CASPT2 or RASPT2 Level of Theory.

\begin{tabular}{lcc}
\hline \hline Active space & MAXD & RMSD \\
\hline & Planar 1,3-butadiene & \\
CAS $(4 \mathrm{e}, 4 \mathrm{o})^{\mathrm{b}}$ & $4.16 \times 10^{-6}$ & $2.38 \times 10^{-6}$ \\
$\mathrm{CAS}(4 \mathrm{e}, 4 \mathrm{o})$ & $1.53 \times 10^{-7}$ & $7.81 \times 10^{-8}$ \\
$\operatorname{RAS}(4 \mathrm{e}, 4 \mathrm{o}) /(0 \mathrm{e}, 0 \mathrm{o}) / 2^{\mathrm{c}}$ & $3.10 \times 10^{-6}$ & $7.23 \times 10^{-7}$ \\
$\operatorname{RAS}(4 \mathrm{e}, 4 \mathrm{o}) /(2 \mathrm{e}, 2 \mathrm{o}) / 1^{\mathrm{c}}$ & $2.59 \times 10^{-7}$ & $1.13 \times 10^{-7}$ \\
& $3.11 \times 10^{-7}$ & $1.43 \times 10^{-7}$ \\
$\mathrm{CAS}(4 \mathrm{e}, 4 \mathrm{o})$ & $4.09 \times 10^{-6}$ & $8.18 \times 10^{-7}$ \\
$\operatorname{RAS}(4 \mathrm{e}, 4 \mathrm{o}) /(0 \mathrm{e}, 0 \mathrm{o}) / 2^{\mathrm{c}}$ & $8.75 \times 10^{-7}$ & $3.29 \times 10^{-7}$ \\
$\operatorname{RAS}(4 \mathrm{e}, 4 \mathrm{o}) /(2 \mathrm{e}, 2 \mathrm{o}) / 1^{\mathrm{c}}$ & Twisted $1,3-$ butadiene & \\
& Naphthalene & $2.68 \times 10^{-6}$ \\
$\mathrm{CAS}(10 \mathrm{e}, 10 \mathrm{o})^{\mathrm{c}}$ & $6.32 \times 10^{-6}$ & $2.75 \times 10^{-6}$ \\
$\operatorname{RAS}(10 \mathrm{e}, 10 \mathrm{o}) /(0 \mathrm{e}, 0 \mathrm{o}) / 2^{\mathrm{c}}$ & $5.79 \times 10^{-6}$ & $5.43 \times 10^{-6}$ \\
$\operatorname{RAS}(10 \mathrm{e}, 10 \mathrm{o}) /(4 \mathrm{e}, 4 \mathrm{o}) / 2^{\mathrm{c}}$ & $9.01 \times 10^{-6}$ & \\
\hline \hline
\end{tabular}

\footnotetext{
${ }^{a}$ Forces perpendicular to the planar axis are not included in RMSD for the planar geometry

${ }^{\mathrm{b}}$ Without the density-fitting approximation

${ }^{c}$ With an imaginary shift of $0.2 \mathrm{i}$
}

\section{B. Performance of RASPT2}

The performance of RASPT2 against CASPT2 was evaluated using trans-1,3,5,7,9,11-dodecahexaene $\left(\mathrm{C}_{12} \mathrm{H}_{14}\right)$. Geometry optimizations were performed with various partitionings of the active space, and the vertical excitation energies $\left(E^{\mathrm{vert}}\right)$ at the $\mathrm{S}_{0}$ minimum, adiabatic excitation energies $\left(E^{\mathrm{AEE}}\right)$, and maximum deviations of the $\mathrm{C}-\mathrm{C}$ bond lengths (as an indicator of the geometrical difference) were calculated. Note that the adiabatic excitation energy is the difference between the energies at the $S_{1}$ and $S_{0}$ minima, and the zero-point vibrational energy (ZPVE) correction was not considered.

Table II shows that the density-fitting approximation does not sacrifice the accuracy, as ob- 
TABLE II. Deviations in Vertical Excitation Energies at the $S_{0}$ minimum ( $E^{\text {vert }}$ in eV), Adiabatic Excitation Energies ( $E^{\mathrm{AEE}}$ in $\mathrm{eV}$ ), and Maximum Absolute Deviations of $\mathrm{C}-\mathrm{C}$ Bond Lengths (Max $\left|\Delta d_{\mathrm{C}-\mathrm{C}}^{\mathrm{S}^{0}}\right|$ and $\operatorname{Max}\left|\Delta d_{\mathrm{C}-\mathrm{C}}^{\mathrm{S}^{1}}\right|$ in $\AA$ ) with Different Partitionings of the Active Space $(12 \mathrm{e}, 12 \mathrm{o})$ for CASPT2 and RASPT2 Calculations.

\begin{tabular}{lcccccc}
\hline \hline Partitioning & $N^{\mathrm{CSF}}$ & $E^{\mathrm{vert}}$ & $E^{\mathrm{AEE}}$ & $\operatorname{Max}\left|\Delta d_{\mathrm{C}-\mathrm{C}}^{\mathrm{S}^{0}}\right|$ & $\operatorname{Max}\left|\Delta d_{\mathrm{C}-\mathrm{C}}^{\mathrm{S}^{1}}\right|$ & $t^{\mathrm{CI} a}$ \\
\hline $\operatorname{CAS}(12 \mathrm{e}, 12 \mathrm{o})^{\mathrm{b}}$ & 226512 & $(3.385)$ & $(2.615)$ & - & - & 459 \\
$\operatorname{CAS}(12 \mathrm{e}, 12 \mathrm{o})$ & 226512 & -0.001 & 0.000 & $5.00 \times 10^{-5}$ & $5.00 \times 10^{-5}$ & 463 \\
$\operatorname{RAS}(12 \mathrm{e}, 12 \mathrm{o}) /(0 \mathrm{e}, 0 \mathrm{o}) / 2$ & 703 & 1.188 & 1.138 & $5.09 \times 10^{-3}$ & $7.04 \times 10^{-3}$ & 1 \\
$\operatorname{RAS}(12 \mathrm{e}, 12 \mathrm{o}) /(0 \mathrm{e}, 0 \mathrm{o}) / 4$ & 28278 & 0.038 & 0.044 & $8.80 \times 10^{-4}$ & $1.39 \times 10^{-3}$ & 41 \\
$\operatorname{RAS}(12 \mathrm{e}, 12 \mathrm{o}) /(0 \mathrm{e}, 0 \mathrm{o}) / 6$ & 147042 & 0.033 & 0.033 & $1.30 \times 10^{-3}$ & $8.50 \times 10^{-4}$ & 289 \\
$\operatorname{RAS}(12 \mathrm{e}, 12 \mathrm{o}) /(2 \mathrm{e}, 2 \mathrm{o}) / 2$ & 2028 & 0.535 & 0.466 & $4.21 \times 10^{-3}$ & $8.35 \times 10^{-3}$ & 3 \\
$\operatorname{RAS}(12 \mathrm{e}, 12 \mathrm{o}) /(4 \mathrm{e}, 4 \mathrm{o}) / 2$ & 8860 & 0.080 & 0.079 & $2.26 \times 10^{-3}$ & $2.76 \times 10^{-3}$ & 16 \\
$\operatorname{RAS}(12 \mathrm{e}, 12 \mathrm{o}) /(6 \mathrm{e}, 6 \mathrm{o}) / 2$ & 36148 & 0.041 & 0.038 & $1.19 \times 10^{-3}$ & $1.47 \times 10^{-3}$ & 61 \\
$\operatorname{RAS}(12 \mathrm{e}, 12 \mathrm{o}) /(8 \mathrm{e}, 8 \mathrm{o}) / 2$ & 115548 & 0.017 & 0.018 & $4.80 \times 10^{-4}$ & $5.90 \times 10^{-4}$ & 238 \\
\hline \hline
\end{tabular}

${ }^{a}$ Wall time for evaluating the partial derivative with respect to the CI coefficient (in seconds).

${ }^{\mathrm{b}}$ Without the density-fitting approximation; $E^{\mathrm{vert}}$ and $E^{\mathrm{AEE}}$ are shown for reference.

served in Ref. 58. In terms of $E^{\mathrm{vert}}$ and $E^{\mathrm{AEE}}$, the deviation is less than $0.001 \mathrm{eV}$, indicating negligible degradation. The difference in bond length is also negligible; the maximum deviation is only $5.00 \times 10^{-5} \AA$.

The number of CSFs $\left(N^{\mathrm{CSF}}\right)$ is greatly reduced by employing RAS references. The computational cost for evaluating the partial derivative of the energy with respect to the CI coefficient $\left[t^{\mathrm{CI}}\right.$; Eq. (17)] is almost proportional to $N^{\mathrm{CSF}}$. Although the use of a very small number of CSFs, such as $\operatorname{RAS}(12 \mathrm{e}, 12 \mathrm{o}) /(0 \mathrm{e}, 0 \mathrm{o}) / 2$ or $\operatorname{RAS}(12 \mathrm{e}, 12 \mathrm{o}) /(2 \mathrm{e}, 2 \mathrm{o}) / 2$, is not appropriate, other RASs give reasonable agreement with the reference CAS result; the deviations in excitation energies and C$\mathrm{C}$ bond lengths are less than $0.1 \mathrm{eV}$ and $5.0 \times 10^{-3} \AA$, respectively. Thus, either more than two electron excitations from RAS1 and into RAS3 or moderate RAS2 spaces should be employed to reasonably reproduce the corresponding CAS result.

The convergence of the SCF and Z-vector equation with the RASs is not as smooth as in the case with CAS; however, analytic derivatives can be efficiently evaluated with larger active spaces. 

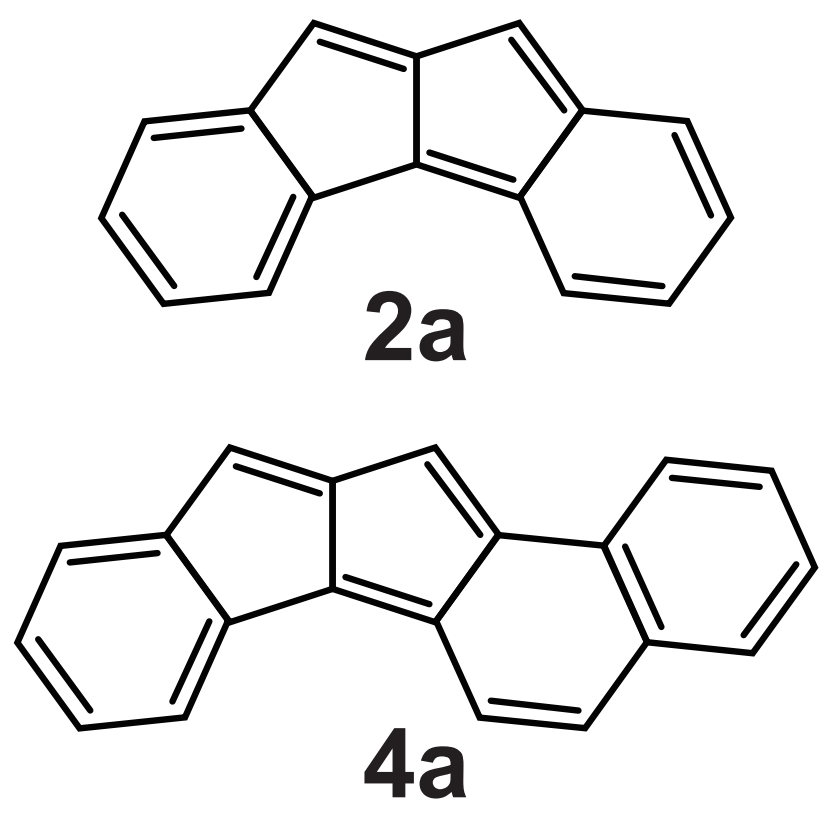

FIG. 2. Molecular structures of 2a and 4a.

\section{Calculations with Larger Active Spaces}

Using the developed method, it is possible to perform geometry optimizations with larger active spaces. As a demonstration, RASSCF and RASPT2 calculations were performed for dibenzopentalene derivatives ${ }^{56} \mathbf{2 a}$ and $\mathbf{4 a}$ (Fig. 2) with active spaces of $\operatorname{RAS}(16 \mathrm{e}, 16 \mathrm{o}) /(4 \mathrm{e}, 4 \mathrm{o}) / 2$ and $\operatorname{RAS}(20 \mathrm{e}, 20 \mathrm{o}) /(4 \mathrm{e}, 4 \mathrm{o}) / 2$, respectively. The initial structures were taken from Ref. 56 and optimized at the RB3LYP-D3/6-311G* level of theory. The adiabatic singlet-triplet energy gaps ( $\Delta E_{\mathrm{ST}}:=\Delta E_{\mathrm{S}}-E_{\mathrm{T}}$ without ZPVE corrections) were then computed. The numerical results are summarized in Table III. The gaps computed with spin-flip noncolinear time-dependent density functional theory (SF-NC-TDDFT/6-311G(d) with the PBE50 functional) in Ref. 56 and at the UB3LYP/6-31G(d,p) and UBLYP/6-31G(d,p) levels of theory in Ref. 59 are also tabulated.

The numbers of CSFs for the RAS(20e,20o)/(4e,4o)/2 partitioning were 110292 and 184863 for the singlet and triplet states, respectively. Although these numbers are smaller than those for CAS $(12 \mathrm{e}, 12 \mathrm{o})$ reported in the previous subsection, the required computational cost was much greater (approximately $t^{\mathrm{CI}}=7700 \mathrm{~s}$ for the triplet state) due to the large number of active orbitals. Still, these calculations were doable on a single computer node. A comparison of RASSCF and RASPT2 indicates that RASSCF clearly overestimates the stability of the singlet state by over $5 \mathrm{kcal} / \mathrm{mol}$. The final energy gap predicted with RASPT2 is similar to that for SF-NC- 
TABLE III. Adiabatic Singlet-Triplet Energy Gaps $\left(\Delta E_{\mathrm{ST}}\right)$ in $\mathrm{kcal} / \mathrm{mol}$ at the RASSCF, RASPT2, SF-NCTDDFT, ${ }^{56}$ UB3LYP, and UBLYP ${ }^{59}$ Levels of Theory.

\begin{tabular}{lcc}
\hline \hline & $\mathbf{2 a}$ & $\mathbf{4 a}$ \\
\hline RASSCF & -6.85 & -15.33 \\
RASPT2 & -1.35 & -8.91 \\
SF-NC-TDDFT $^{56}$ & 2.48 & -8.20 \\
UB3LYP $^{59}$ & 0.23 & - \\
UBLYP $^{59}$ & -1.50 & - \\
\hline \hline
\end{tabular}

TDDFT/6-311G(d) (a difference of $3.83 \mathrm{kcal} / \mathrm{mol}$ ), although the RASSCF and RASPT2 calculations predicted the singlet state to be more stable than the triplet state for $\mathbf{2 a}$, in agreement with the UBLYP/6-31G(d,p) calculations. However, the energy gap is very small, making it difficult to determine the ground state. Even CASPT2 sometimes produces an error of $0.2-0.3 \mathrm{eV}(\sim 5$ $\mathrm{kcal} / \mathrm{mol}$ ) relative to experimental results. ${ }^{60}$

In Ref. 56, the open-shell character is discussed with the diradical character, which was obtained from the occupation number of the lowest unoccupied natural orbitals. ${ }^{56}$ For multiconfiguration methods, the open-shell character may be discussed based on the weight of the configuration. For the ground states of $\mathbf{2 a}$ and $\mathbf{4 a}$, the contributions of the closed-shell configuration are $32 \%$ and $37 \%$, respectively. Although the closed-shell contribution is slightly greater for $\mathbf{4 a}$, the difference is small, and both derivatives have strong multiconfiguration character.

The presented result for $\Delta E_{\mathrm{ST}}$ is not a definitive prediction. Considering the dependences of the computed properties on the size of the active space, the accuracy may not be satisfactory with the present definition of the RAS. Based on the error analysis presented in Table II, the present numerical result may deviate from the CAS(20e,20o) result by a few $\mathrm{kcal} / \mathrm{mol}$. In addition, the use of the state-averaged density matrix in the Fock matrix may cause an observable difference. ${ }^{47,48}$ Nevertheless, the present numerical result demonstrates that geometry optimizations with the (20e,20o) active space can be performed using MRPT, and the predicted gap is rather reasonable. 


\section{CONCLUSIONS}

Analytic gradients of the single-state RASPT2 as well as CASPT2 methods were developed and

implemented in a local version of OpenMolcas. Similar to previous CASPT2 developments, ${ }^{16,17,19}$ the responses of the wavefunction parameters were evaluated by solving one linear Z-vector equation. The correctness of the implementation was shown by comparison with numerical gradients. The performance of RASPT2 against CASPT2 was also investigated, demonstrating that a sensible partitioning of the active space in RASPT2 reproduces the corresponding CASPT2 result well. The present implementation can use active spaces with large sizes up to (20e,20o); however, the RAS2 space and the maximum number of holes and electrons cannot be large.

CASPT2 and RASPT2 are not size-consistent. The properties that are computed in this study are not substantially affected by their size inconsistency, because a single molecule is targeted. However, non-unimolecular properties such as binding energies may be affected by this inconsistency; thus, a special attention is required. According to Ref. 61, the size inconsistency of internally contracted CASPT2 seems to be small, but the error of RASPT2 may be larger and should be carefully evaluated. In the future, the use of the state-specific density matrix in the Fock matrix and (extended) ${ }^{35}$ multistate extension ${ }^{62}$ will be considered, including conical intersection search as in Refs. 21, 39, 40, and 63. Moreover, algorithmic improvement is essential for practical use. The present implementation cannot use a large number of atomic orbitals $(\sim 400)$.

\section{SUPPLEMENTARY MATERIAL}

See the supplementary material for the optimized coordinates of trans-1,3,5,7,9,11-dodecahexaene and dibenzopentalene derivatives.

\section{ACKNOWLEDGMENTS}

I wish to acknowledge the kind support from Professors Hayashi and Kurashige (Kyoto University). This work was supported by JSPS KAKENHI Grant Number 20K15230. 


\section{DATA AVAILABILITY}

The data that support the findings of this study are available from the corresponding author upon reasonable request.

\section{REFERENCES}

${ }^{1}$ M. Musiał, A. Perera, and R. J. Bartlett, "Multireference coupled-cluster theory: The easy way," J. Chem. Phys. 134, 114108 (2011).

${ }^{2}$ D. I. Lyakh, M. Musiał, V. F. Lotrich, and R. J. Bartlett, "Multireference nature of chemistry: The coupled-cluster view," Chem. Rev. 112, 182-243 (2012).

${ }^{3}$ A. Köhn, M. Hanauer, L. A. Mück, T.-C. Jagau, and J. Gauss, "State-specific multireference coupled-cluster theory,” WIREs Comput. Mol. Sci. 3, 176-197 (2013).

${ }^{4}$ P. G. Szalay, T. Müller, G. Gidofalvi, H. Lischka, and R. Shepard, "Multiconfiguration selfconsistent field and multireference configuration interaction methods and applications," Chem. Rev. 112, 108-181 (2012).

${ }^{5}$ B. O. Roos, P. Linse, P. E. Siegbahn, and M. R. Blomberg, "A simple method for the evaluation of the second-order-perturbation energy from external double-excitations with a CASSCF reference wavefunction," Chem. Phys. 66, 197 - 207 (1982).

${ }^{6}$ K. Andersson, P.-Å. Malmqvist, B. O. Roos, A. J. Sadlej, and K. Wolinski, "Second-order perturbation theory with a CASSCF reference function,” J. Phys. Chem. 94, 5483-5488 (1990). ${ }^{7}$ K. Andersson, P.-Å. Malmqvist, and B. O. Roos, "Second-order perturbation theory with a complete active space self-consistent field reference function,” J. Chem. Phys. 96, 1218-1226 (1992).

${ }^{8}$ A. A. Granovsky, "Extended multi-configuration quasi-degenerate perturbation theory: The new approach to multi-state multi-reference perturbation theory,' J. Chem. Phys. 134, 214113 (2011).

${ }^{9}$ H. Nakano, “Quasidegenerate perturbation theory with multiconfigurational self-consistent-field reference functions," J. Chem. Phys. 99, 7983-7992 (1993).

${ }^{10}$ C. Angeli, R. Cimiraglia, S. Evangelisti, T. Leininger, and J.-P. Malrieu, "Introduction of $n$-electron valence states for multireference perturbation theory," J. Chem. Phys. 114, 1025210264 (2001). 
${ }^{11}$ C. Angeli, R. Cimiraglia, and J.-P. Malrieu, " $n$-electron valence state perturbation theory: a fast implementation of the strongly contracted variant," Chem. Phys. Lett. 350, 297-305 (2001).

${ }^{12}$ C. Angeli, R. Cimiraglia, and J.-P. Malrieu, " $n$-electron valence state perturbation theory: A spinless formulation and an efficient implementation of the strongly contracted and of the partially contracted variants," J. Chem. Phys. 117, 9138-9153 (2002).

${ }^{13}$ Y. G. Khait, J. Song, and M. R. Hoffmann, "Explication and revision of generalized van vleck perturbation theory for molecular electronic structure," J. Chem. Phys. 117, 4133-4145 (2002).

${ }^{14}$ R. F. Fink, "Two new unitary-invariant and size-consistent perturbation theoretical approaches to the electron correlation energy," Chem. Phys. Lett. 428, 461-466 (2006).

${ }^{15}$ J. W. Park, R. Al-Saadon, M. K. MacLeod, T. Shiozaki, and B. Vlaisavljevich, "Multireference electron correlation methods: Journeys along potential energy surfaces," Chem. Rev. 120, 58785909 (2020).

${ }^{16}$ P. Celani and H.-J. Werner, “Analytical energy gradients for internally contracted second-order multireference perturbation theory," J. Chem. Phys. 119, 5044-5057 (2003).

${ }^{17}$ M. K. MacLeod and T. Shiozaki, "Communication: Automatic code generation enables nuclear gradient computations for fully internally contracted multireference theory,' J. Chem. Phys. 142, 051103 (2015).

${ }^{18}$ Y. Nishimoto, "Analytic first-order derivatives of partially contracted n-electron valence state second-order perturbation theory (PC-NEVPT2)," J. Chem. Phys. 151, 114103 (2019).

${ }^{19}$ C. Song, J. B. Neaton, and T. J. Martínez, "Reduced scaling formulation of CASPT2 analytical gradients using the supporting subspace method," J. Chem. Phys. 154, 014103 (2021).

${ }^{20}$ J. W. Park and T. Shiozaki, "On-the-fly CASPT2 surface-hopping dynamics,” J. Chem. Theory Comput. 13, 3676-3683 (2017).

${ }^{21}$ J. W. Park, “Analytical gradient theory for quasidegenerate n-electron valence state perturbation theory (QD-NEVPT2)," J. Chem. Theory Comput. 16, 326-339 (2020).

${ }^{22}$ P.-Å. Malmqvist, A. Rendell, and B. O. Roos, “The restricted active space self-consistent-field method, implemented with a split graph unitary group approach,” J. Phys. Chem. 94, 5477-5482 (1990).

${ }^{23}$ P. Celani and H.-J. Werner, "Multireference perturbation theory for large restricted and selected active space reference wave functions," J. Chem. Phys. 112, 5546-5557 (2000).

${ }^{24}$ P.-Å. Malmqvist, K. Pierloot, A. R. M. Shahi, C. J. Cramer, and L. Gagliardi, “The restricted active space followed by second-order perturbation theory method: Theory and application to 
the study of $\mathrm{CuO}_{2}$ and $\mathrm{Cu}_{2} \mathrm{O}_{2}$ systems," J. Chem. Phys. 128, 204109 (2008).

${ }^{25}$ H. Nakano, J. Nakatani, and K. Hirao, "Second-order quasi-degenerate perturbation theory with quasi-complete active space self-consistent field reference functions," J. Chem. Phys. 114, 1133-1141 (2001).

${ }^{26}$ H. Nakano, R. Uchiyama, and K. Hirao, "Quasi-degenerate perturbation theory with general multiconfiguration self-consistent field reference functions," J. Comput. Chem. 23, 1166-1175 (2002).

${ }^{27}$ L. Roskop and M. S. Gordon, "Quasi-degenerate second-order perturbation theory for occupation restricted multiple active space self-consistent field reference functions," J. Chem. Phys. 135, 044101 (2011).

${ }^{28}$ Y. Kurashige and T. Yanai, "Second-order perturbation theory with a density matrix renormalization group self-consistent field reference function: Theory and application to the study of chromium dimer," J. Chem. Phys. 135, 094104 (2011).

${ }^{29}$ J. W. Park, R. Al-Saadon, N. E. Strand, and T. Shiozaki, "Imaginary shift in CASPT2 nuclear gradient and derivative coupling theory,' J. Chem. Theory Comput. 15, 4088-4098 (2019).

${ }^{30}$ B. O. Roos and K. Andersson, "Multiconfigurational perturbation theory with level shift - the $\mathrm{Cr}_{2}$ potential revisited," Chem. Phys. Lett. 245, 215-223 (1995).

${ }^{31}$ N. Forsberg and P.- $\AA$. Malmqvist, "Multiconfiguration perturbation theory with imaginary level shift," Chem. Phys. Lett. 274, 196-204 (1997).

${ }^{32}$ F. Menezes, D. Kats, and H.-J. Werner, "Local complete active space second-order perturbation theory using pair natural orbitals (PNO-CASPT2),” J. Chem. Phys. 145, 124115 (2016).

${ }^{33}$ T. Helgaker, S. Coriani, P. Jørgensen, K. Kristensen, J. Olsen, and K. Ruud, "Recent advances in wave function-based methods of molecular-property calculations," Chem. Rev. 112, 543-631 (2012).

${ }^{34}$ N. C. Handy and H. F. Schaefer III, "On the evaluation of analytic energy derivatives for correlated wave functions," J. Chem. Phys. 81, 5031-5033 (1984).

${ }^{35}$ T. Shiozaki, W. Győrffy, P. Celani, and H.-J. Werner, "Communication: Extended multi-state complete active space second-order perturbation theory: Energy and nuclear gradients," J. Chem. Phys. 135, 081106 (2011).

${ }^{36}$ K. L. Bak, J. Boatz, and J. Simons, "First-order geometrical response equations for stateaveraged multiconfigurational self-consistent field (SA-MCSCF) wave functions,' Int. J. Quantum Chem. 40, 361-378 (1991). 
${ }^{37}$ J. Stålring, A. Bernhardsson, and R. Lindh, "Analytical gradients of a state average MCSCF state and a state average diagnostic," Mol. Phys. 99, 103-114 (2001).

${ }^{38}$ I. Fdez. Galván, M. G. Delcey, T. B. Pedersen, F. Aquilante, and R. Lindh, "Analytical stateaverage complete-active-space self-consistent field nonadiabatic coupling vectors: Implementation with density-fitted two-electron integrals and application to conical intersections," J. Chem. Theory Comput. 12, 3636-3653 (2016).

${ }^{39}$ J. W. Park and T. Shiozaki, "Analytical derivative coupling for multistate CASPT2 theory," J. Chem. Theory Comput. 13, 2561-2570 (2017).

${ }^{40}$ Y. Nishimoto, "Locating conical intersections using the quasidegenerate partially and strongly contracted NEVPT2 methods," Chem. Phys. Lett. 744, 137219 (2020).

${ }^{41}$ J. W. Park, "Analytical gradient theory for strongly contracted (SC) and partially contracted (PC) n-electron valence state perturbation theory (NEVPT2)," J. Chem. Theory Comput. 15, 5417-5425 (2019).

${ }^{42}$ R. Moccia, "Variable bases in SCF MO calculations," Chem. Phys. Lett. 5, 260-264 (1970).

${ }^{43}$ N. Handy, R. Amos, J. Gaw, J. Rice, and E. Simandiras, "The elimination of singularities in derivative calculations," Chem. Phys. Lett. 120, 151-158 (1985).

${ }^{44}$ I. Fdez. Galván, M. Vacher, A. Alavi, C. Angeli, F. Aquilante, J. Autschbach, J. J. Bao, S. I. Bokarev, N. A. Bogdanov, R. K. Carlson, L. F. Chibotaru, J. Creutzberg, N. Dattani, M. G. Delcey, S. S. Dong, A. Dreuw, L. Freitag, L. M. Frutos, L. Gagliardi, F. Gendron, A. Giussani, L. González, G. Grell, M. Guo, C. E. Hoyer, M. Johansson, S. Keller, S. Knecht, G. Kovačević, E. Källman, G. Li Manni, M. Lundberg, Y. Ma, S. Mai, J. P. Malhado, P.-Å. Malmqvist, P. Marquetand, S. A. Mewes, J. Norell, M. Olivucci, M. Oppel, Q. M. Phung, K. Pierloot, F. Plasser, M. Reiher, A. M. Sand, I. Schapiro, P. Sharma, C. J. Stein, L. K. Sørensen, D. G. Truhlar, M. Ugandi, L. Ungur, A. Valentini, S. Vancoillie, V. Veryazov, O. Weser, T. A. Wesołowski, P.-O. Widmark, S. Wouters, A. Zech, J. P. Zobel, and R. Lindh, "OpenMolcas: From source code to insight," J. Chem. Theory Comput. 15, 5925-5964 (2019).

${ }^{45}$ F. Aquilante, J. Autschbach, A. Baiardi, S. Battaglia, V. A. Borin, L. F. Chibotaru, I. Conti, L. De Vico, M. Delcey, I. Fdez. Galván, N. Ferré, L. Freitag, M. Garavelli, X. Gong, S. Knecht, E. D. Larsson, R. Lindh, M. Lundberg, P. Å. Malmqvist, A. Nenov, J. Norell, M. Odelius, M. Olivucci, T. B. Pedersen, L. Pedraza-González, Q. M. Phung, K. Pierloot, M. Reiher, I. Schapiro, J. Segarra-Martí, F. Segatta, L. Seijo, S. Sen, D.-C. Sergentu, C. J. Stein, L. Ungur, M. Vacher, A. Valentini, and V. Veryazov, "Modern quantum chemistry with [Open]Molcas," J. 
Chem. Phys. 152, 214117 (2020).

${ }^{46}$ J. Olsen, D. L. Yeager, and P. Jørgensen, “Optimization and characterization of a multiconfigurational self-consistent field (MCSCF) state," in Advances in Chemical Physics (John Wiley \& Sons, Ltd, 1983) pp. 1-176.

${ }^{47}$ J. W. Park, "Single-state single-reference and multistate multireference zeroth-order hamiltonians in MS-CASPT2 and conical intersections," J. Chem. Theory Comput. 15, 3960-3973 (2019).

${ }^{48}$ D. Kats and H.-J. Werner, "Multi-state local complete active space second-order perturbation theory using pair natural orbitals (PNO-MS-CASPT2)," J. Chem. Phys. 150, 214107 (2019).

${ }^{49}$ S. Gozem, F. Melaccio, A. Valentini, M. Filatov, M. Huix-Rotllant, N. Ferré, L. M. Frutos, C. Angeli, A. I. Krylov, A. A. Granovsky, R. Lindh, and M. Olivucci, "Shape of multireference, equation-of-motion coupled-cluster, and density functional theory potential energy surfaces at a conical intersection,” J. Chem. Theory Comput. 10, 3074-3084 (2014).

${ }^{50}$ T. H. Dunning, "Gaussian basis sets for use in correlated molecular calculations. I. The atoms boron through neon and hydrogen," J. Chem. Phys. 90, 1007-1023 (1989).

${ }^{51}$ R. A. Kendall, T. H. Dunning, and R. J. Harrison, "Electron affinities of the first-row atoms revisited. Systematic basis sets and wave functions," J. Chem. Phys. 96, 6796-6806 (1992).

${ }^{52}$ D. Feller, "The role of databases in support of computational chemistry calculations," J. Comput. Chem. 17, 1571-1586 (1996).

${ }^{53}$ K. L. Schuchardt, B. T. Didier, T. Elsethagen, L. Sun, V. Gurumoorthi, J. Chase, J. Li, and T. L. Windus, "Basis set exchange: A community database for computational sciences," J. Chem. Inf. Model. 47, 1045-1052 (2007).

${ }^{54}$ B. P. Pritchard, D. Altarawy, B. Didier, T. D. Gibson, and T. L. Windus, "New basis set exchange: An open, up-to-date resource for the molecular sciences community," J. Chem. Inf. Model. 59, 4814-4820 (2019).

${ }^{55}$ G. Ghigo, B. O. Roos, and P.- $\AA$. Malmqvist, "A modified definition of the zeroth-order hamiltonian in multiconfigurational perturbation theory (CASPT2)," Chem. Phys. Lett. 396, 142 - 149 (2004).

${ }^{56}$ A. Konishi, Y. Okada, R. Kishi, M. Nakano, and M. Yasuda, "Enhancement of antiaromatic character via additional benzoannulation into dibenzo[a,f]pentalene: Syntheses and properties of benzo[a]naphtho[2,1-f]pentalene and dinaphtho[2,1-a,f]pentalene,” J. Am. Chem. Soc. 141, 560-571 (2019). 
${ }^{57}$ M. R. Hoffmann, D. J. Fox, J. F. Gaw, Y. Osamura, Y. Yamaguchi, R. S. Grev, G. Fitzgerald, H. F. Schaefer, P. J. Knowles, and N. C. Handy, "Analytic energy second derivatives for general MCSCF wave functions,” J. Chem. Phys. 80, 2660-2668 (1984).

${ }^{58}$ W. Győrffy, T. Shiozaki, G. Knizia, and H.-J. Werner, "Analytical energy gradients for secondorder multireference perturbation theory using density fitting," J. Chem. Phys. 138, 104104 (2013).

${ }^{59}$ A. Konishi, Y. Okada, M. Nakano, K. Sugisaki, K. Sato, T. Takui, and M. Yasuda, "Synthesis and characterization of dibenzo[a,f]pentalene: Harmonization of the antiaromatic and singlet biradical character,” J. Am. Chem. Soc. 139, 15284-15287 (2017).

${ }^{60}$ J. P. Zobel, J. J. Nogueira, and L. González, “The IPEA dilemma in CASPT2,” Chem. Sci. 8, 1482-1499 (2017).

${ }^{61}$ J. M. Rintelman, I. Adamovic, S. Varganov, and M. S. Gordon, "Multireference second-order perturbation theory: How size consistent is " almost size consistent"," J. Chem. Phys. 122, 044105 (2005).

${ }^{62}$ J. Finley, P.-Å. Malmqvist, B. O. Roos, and L. Serrano-Andrés, “The multi-state CASPT2 method," Chem. Phys. Lett. 288, 299-306 (1998).

${ }^{63}$ J. W. Park, "Analytical first-order derivatives of second-order extended multiconfiguration quasidegenerate perturbation theory (XMCQDPT2): Implementation and application,' J. Chem. Theory Comput. 16, 5562-5571 (2020). 